\title{
ON THE EULER CHARACTERISTIC OF GENERALIZED KUMMER VARIETIES
}

\section{Olivier Debarre ${ }^{1}$}

The aim of this note is to apply the Yau-Zaslow-Beauville method ([YZ], [B1]) to compute the Euler characteristic of the generalized Kummer varieties attached to a complex abelian surface (a calculation also done in [GS] by different methods). The argument is very geometric : given an ample line bundle $\mathrm{L}$ with $h^{0}(\mathrm{~L})=n$ on an abelian surface A, such that each curve in $|\mathrm{L}|$ is integral, we construct a projective symplectic $(2 n-2)$ dimensional variety $\mathrm{J}^{d}(\mathrm{~A})$ with a Lagrangian fibration $\mathrm{J}^{d}(\mathrm{~A}) \rightarrow|\mathrm{L}|$ whose fiber over a point corresponding to a smooth curve $\mathrm{C}$ is the kernel of the Albanese map $\mathrm{J}^{d} \mathrm{C} \rightarrow \mathrm{A}$. The Yau-Zaslow-Beauville's method shows that the Euler characteristic of $\mathrm{J}^{d}(\mathrm{~A})$ is $n$ times the number of genus 2 curves in $|\mathrm{L}|$, to wit $n^{2} \sigma(n)$ (where $\sigma(n)=\sum_{m \mid n} m$ ). The latter computation was also done in $[\mathrm{G}]$, where a general conjecture (proved in [BL] for K3 surfaces) expresses these numbers in terms of quasi-modular forms : if $\mathrm{N}_{r}^{n}$ is the number of genus $r+2$ curves in $|\mathrm{L}|$ passing through $r$ general points of $\mathrm{A}$, one should have

$$
\sum_{n \in \mathbf{N}} \mathrm{N}_{r}^{n} q^{n}=\left(\sum_{n \in \mathbf{N}} n \sigma(n) q^{n}\right)^{r}\left(\sum_{n \in \mathbf{N}} n^{2} \sigma(n) q^{n}\right) .
$$

Unlike the case of K3 surfaces, none of these varieties $\mathrm{J}^{d}(\mathrm{~A})$ seem to be birationally isomorphic to the generalized Kummer $\mathrm{K}_{n-1}(\mathrm{~A})$ introduced by Beauville in [B2] (a symplectic desingularization of a fiber of the sum morphism $\left.\mathrm{A}^{(n)} \rightarrow \mathrm{A}\right)$. However, we check that this is the case when $\mathrm{A}$ is a product of elliptic curves and $d=n-2$. Using a result of Huybrechts, we conclude that $\mathrm{K}_{n-1}(\mathrm{~A})$ and $\mathrm{J}^{n-2}(\mathrm{~A})$ are diffeomorphic hence have the same Euler characteristic $n^{3} \sigma(n)$. I would like to thank D. Huybrechts very much for his help with theorem 3.4 .

\section{The symplectic variety $\mathrm{J}^{d}(\mathrm{~A})$}

Let $\mathrm{A}$ be a complex abelian surface with a polarization $\ell$ of type $(1, n)$. Assume that each curve with class $\ell$ is integral (this holds for generic $(\mathrm{A}, \ell)$ ). Let $\hat{\mathrm{A}}$ be the dual abelian surface. Let $\phi_{\ell}: \mathrm{A} \rightarrow \hat{\mathrm{A}}$ be the morphism associated with the polarization $\ell$; there exists a factorization $n \operatorname{Id}_{\mathrm{A}}: \mathrm{A} \stackrel{\phi_{\ell}}{\longrightarrow} \hat{\mathrm{A}} \stackrel{\phi_{\hat{\ell}}}{\longrightarrow} \mathrm{A}$, where $\hat{\ell}$ is a polarization on $\hat{\mathrm{A}}$ of type $(1, n)$.

We denote by $\operatorname{Pic}^{\ell}(\mathrm{A})$ the component of the Picard group of A corresponding to line bundles with class $\ell$, by $\{\ell\}$ the component of the Hilbert scheme that parametrizes curves in A with class $\ell$, by $\mathcal{C} \rightarrow\{\ell\}$ the universal family, and by $\overline{\mathcal{J}} \mathcal{C} \rightarrow\{\ell\}$ the compactified Picard scheme of this family $([\mathrm{AK}])$.

The variety $\overline{\mathcal{J}} \mathcal{C}$ splits as a disjoint union $\coprod_{d \in \mathbf{z}} \overline{\mathcal{J}}^{d} \mathcal{C}$, where $\overline{\mathcal{J}}^{d} \mathcal{C}$ is a projective variety of dimension $2 n+2$, which parameterizes pairs $(\mathrm{C}, \mathcal{L})$ where $\mathrm{C}$ is a curve on $\mathrm{A}$ with class $\ell$ and $\mathcal{L}$ is a torsion free, rank 1 coherent sheaf on $\mathrm{C}$ of degree $d$ (i.e. with $\chi(\mathcal{L})=d+1-g(\mathrm{C})=d-n)$. According to Mukai $([\mathrm{M} 1]$, ex. 0.5$), \overline{\mathcal{J}}^{d} \mathcal{C}$ can be viewed as a connected component of the moduli space of simple sheaves $\mathcal{L}$ on $\mathrm{A}$, and therefore is smooth, and admits a (holomorphic) symplectic structure. There is a natural morphism

$$
\begin{aligned}
& \alpha: \overline{\mathcal{J}}^{d} \mathcal{C} \longrightarrow\{\ell\} \quad \longrightarrow \quad \operatorname{Pic}^{\ell}(\mathrm{A}) \\
& (\mathrm{C}, \mathcal{L}) \longmapsto \mathrm{C} \longmapsto\left[\mathcal{O}_{\mathrm{A}}(\mathrm{C})\right]
\end{aligned}
$$

1 Partially supported by the European HCM Project "Algebraic Geometry in Europe" (AGE), Contract CHRXCT-940557. 
also defined by $\alpha(\mathcal{L})=\operatorname{det} \mathcal{L}$. For each smooth curve $\mathrm{C}$ in $\{\ell\}$, the inclusion $\mathrm{C} \subset \mathrm{A}$ induces an Abel-Jacobi map $\mathrm{J}^{d} \mathrm{C} \rightarrow \mathrm{A}$; this defines a rational map

$$
\beta: \overline{\mathcal{J}}^{d} \mathcal{C} \rightarrow \mathrm{A} \times\{\ell\} \longrightarrow \mathrm{A}
$$

which is regular since $\mathrm{A}$ is an abelian variety and $\overline{\mathcal{J}}^{d} \mathcal{C}$ is normal. Let $\mathrm{J}^{d}(\mathrm{~A})$ be a fiber of the map $(\alpha, \beta): \overline{\mathcal{J}}^{d} \mathcal{C} \longrightarrow \operatorname{Pic}^{\ell}(\mathrm{A}) \times \mathrm{A}$ (they are all isomorphic). Note that $\mathrm{J}^{d}(\mathrm{~A}), \mathrm{J}^{d+2 n}(\mathrm{~A})$ and $\mathrm{J}^{-d}(\mathrm{~A})$ are isomorphic.

Proposition 1.1.- The symplectic structure on $\overline{\mathcal{J}}^{d} \mathcal{C}$ induces a symplectic structure on the $(2 n-2)$-dimensional variety $\mathrm{J}^{d}(\mathrm{~A})$.

Proof. Recall that there is a canonical isomorphism $\mathrm{T}_{\mathcal{L}} \overline{\mathcal{J}}^{d} \mathcal{C} \simeq \operatorname{Ext}^{1}(\mathcal{L}, \mathcal{L})$, and that the symplectic form $\omega$ is the pairing

$$
\operatorname{Ext}^{1}(\mathcal{L}, \mathcal{L}) \otimes \operatorname{Ext}^{1}(\mathcal{L}, \mathcal{L}) \rightarrow \operatorname{Ext}^{2}(\mathcal{L}, \mathcal{L}) \stackrel{\operatorname{Tr}}{\longrightarrow} \mathrm{H}^{2}\left(\mathrm{~A}, \mathcal{O}_{\mathrm{A}}\right) \simeq \mathbf{C}
$$

The map $\mathrm{T}_{\mathcal{L}} \alpha$ is the trace map $\mathrm{T}: \operatorname{Ext}^{1}(\mathcal{L}, \mathcal{L}) \rightarrow \mathrm{H}^{1}\left(\mathrm{~A}, \mathcal{O}_{\mathrm{A}}\right)$, whereas the tangent map at the origin to the map $\iota: \operatorname{Pic}^{0}(\mathrm{~A}) \rightarrow \overline{\mathcal{J}}^{d} \mathcal{C}$ defined by $\iota(\mathrm{P})=\mathrm{P} \otimes \mathcal{L}$ is the dual $\mathrm{T}^{*}: \mathrm{H}^{1}\left(\mathrm{~A}, \mathcal{O}_{\mathrm{A}}\right) \rightarrow \operatorname{Ext}^{1}(\mathcal{L}, \mathcal{L})$. Since $\alpha \iota$ is constant, $\mathrm{T} \circ \mathrm{T}^{*}=0 ;$ in particular

$$
\operatorname{Ker} \mathrm{T} \supset \operatorname{Im}^{*}=(\operatorname{Ker} \mathrm{T})^{\perp}
$$

Note also that $\beta \iota \phi_{\ell}=n \operatorname{Id}_{\mathrm{A}}$ (use the Morikawa-Matsusaka endomorphism), hence $\mathrm{T}_{\mathcal{L}} \beta \circ \mathrm{T}^{*}=\mathrm{T} \phi_{\hat{\ell}}$ and $\operatorname{Ker} \mathrm{T}_{\mathcal{L}} \beta \cap \operatorname{Im} \mathrm{T}^{*}=\{0\}$. Since both $\operatorname{Ker} \mathrm{T}$ and $\operatorname{Ker} \mathrm{T}_{\mathcal{L}} \beta$ have codimension 2 , this implies

$$
\operatorname{Ker} \mathrm{T}=\left(\operatorname{Ker} \mathrm{T} \cap \operatorname{Ker} \mathrm{T}_{\mathcal{L}} \beta\right) \oplus(\operatorname{Ker} \mathrm{T})^{\perp}
$$

and the restriction of $\omega$ to $\operatorname{Ker} T \cap \operatorname{Ker} \mathrm{T}_{\mathcal{L}} \beta=\mathrm{T}_{\mathcal{L}} \mathrm{J}^{d}(\mathrm{~A})$ is non-degenerate.

The map $\alpha$ restricts to a morphism $\alpha: \mathrm{J}^{d}(\mathrm{~A}) \rightarrow|\mathrm{L}|$ whose fiber $\mathrm{K}^{d}(\mathrm{C})$ over the point corresponding to a smooth curve $\mathrm{C}$ is the (connected) kernel of the Abel-Jacobi map $\beta: \mathrm{J}^{d} \mathrm{C} \rightarrow \mathrm{A}$; it is a Lagrangian fibration.

\section{The Euler characteristic of $\mathrm{J}^{d}(\mathrm{~A})$}

We calculate the Euler characteristic of $\mathrm{J}^{d}(\mathrm{~A})$ by using the Lagrangian fibration $\alpha: \mathrm{J}^{d}(\mathrm{~A}) \rightarrow|\mathrm{L}|$, as in $[\mathrm{B} 1]$.

Proposition 2.1.- Let $\mathrm{C}$ be an integral element of $|\mathrm{L}|$. The Euler characteristic of $\mathrm{K}^{d}(\mathrm{C})$ is $n$ if the normalization of $\mathrm{C}$ has genus 2 , and 0 otherwise.

Proof. Let $\eta: \widetilde{\mathrm{C}} \rightarrow \mathrm{C}$ be the normalization. There is a commutative diagram (as in $\S 2$ of $[\mathrm{B} 1]$, we may restrict ourselves to the case $d=0$ )

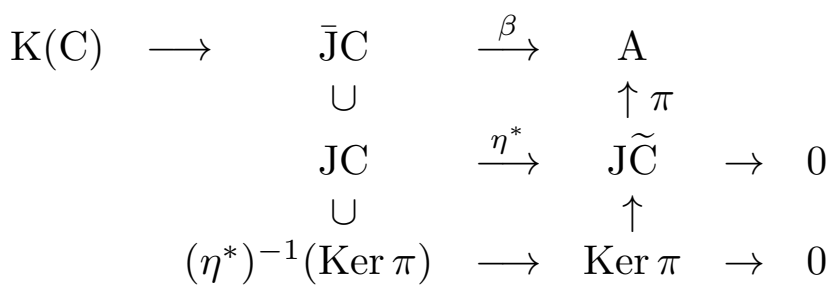


By lemma 2.1 of loc.cit., the group JC acts freely on $\overline{\mathrm{JC}}$. Note also that for $\mathrm{M}$ in JC and $\mathcal{L}$ in $\overline{\mathrm{J}} \mathrm{C}$,

$$
\beta(\mathrm{M} \otimes \mathcal{L})=\beta(\mathrm{M})+\beta(\mathcal{L})
$$

because this is true when $\mathcal{L}$ is invertible, and $\mathrm{JC}$ is dense in $\overline{\mathrm{J} C}$. It follows that $\left(\eta^{*}\right)^{-1}(\operatorname{Ker} \pi)$ acts (freely) on $\mathrm{K}(\mathrm{C})$. As in prop. 2.2 of loc.cit., it follows that $e(\mathrm{~K}(\mathrm{C}))=0$ if $\operatorname{Ker} \pi$ is infinite, that is if $g(\widetilde{\mathrm{C}})>2$.

Assume now that $\widetilde{\mathrm{C}}$ has genus 2 . The situation here is much simpler than in loc.cit., because the normalization $\eta$ of $\mathrm{C}$ is unramified: it is the restriction to $\widetilde{\mathrm{C}}$ of the isogeny $\pi: \mathrm{J} \widetilde{\mathrm{C}} \rightarrow \mathrm{A}$. If $\check{\mathrm{C}} \rightarrow \mathrm{C}$ is the minimal unibranch partial normalization (cf. loc.cit.), it follows that $\widetilde{\mathrm{C}} \rightarrow \check{\mathrm{C}}$ is an unramified homeomorphism, hence an isomorphism (EGA IV, 18.12.6).

There is a commutative diagram

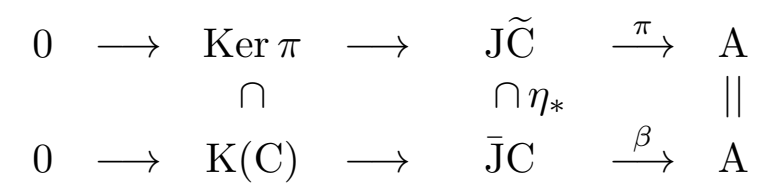

and an exact sequence

$$
1 \rightarrow \mathcal{O}_{\widetilde{\mathrm{C}}}^{*} / \mathcal{O}_{\mathrm{C}}^{*} \longrightarrow \mathrm{JC} \longrightarrow \mathrm{J} \widetilde{\mathrm{C}} \rightarrow 0
$$

If one chooses a line bundle $\mathrm{M}$ on $\mathrm{C}$ corresponding to a point of $\mathcal{O}_{\widetilde{\mathrm{C}}}^{*} / \mathcal{O}_{\mathrm{C}}^{*}$ as in the proof of prop. 3.3 of loc.cit., it acts on $\overline{\mathrm{J}} \mathrm{C}$, hence on $\mathrm{K}(\mathrm{C})$. Beauville's reasoning proves that $\mathrm{M}$ acts freely on the complement of $\eta_{*} \mathrm{~J} \widetilde{\mathrm{C}}$ in $\overline{\mathrm{J} C}$, hence also on the complement of Ker $\pi$ in $\mathrm{K}(\mathrm{C})$. It follows that $e(\mathrm{~K}(\mathrm{C}))=e(\operatorname{Ker} \pi)=n$.

As a corollary, we get, assuming that each curve with class $\ell$ is integral,

$$
e\left(\mathrm{~J}^{d}(\mathrm{~A})\right)=n \operatorname{Card}\{\mathrm{C} \in|\mathrm{L}| \mid g(\widetilde{\mathrm{C}})=2\} .
$$

It remains to count the number of (integral) genus 2 curves $\mathrm{C}$ in $|\mathrm{L}|$. The normalization $\eta: \widetilde{\mathrm{C}} \rightarrow \mathrm{C}$ induces an isogeny $\pi: \mathrm{J} \widetilde{\mathrm{C}} \rightarrow \mathrm{A}$ such that $\pi_{*} \widetilde{\mathrm{C}} \in|\mathrm{L}|$. Let $r$ be the degree of $\pi$; then $\pi^{*} \mathrm{~L}$ is numerically equivalent to $r \widetilde{\mathrm{C}}$, hence $r \mathrm{~L}^{2}=r^{2} \widetilde{\mathrm{C}}^{2}=2 r^{2}$ and $r=n$, and $\pi^{*} \ell$ has type $(n, n)$.

The number of isomorphism classes of isogenies $\pi: \widetilde{\mathrm{A}} \rightarrow \mathrm{A}$ such that $\pi^{*} \ell$ is of type $(n, n)$ is also the number of isomorphism classes of isogenies $\hat{\pi}: \hat{\mathrm{A}} \rightarrow \widetilde{\mathrm{A}}$ where $\widetilde{\mathrm{A}}$ has a principal polarization $\theta$ such that $\hat{\pi}^{*} \theta=\hat{\ell}$, hence also the number of subgroups of $\operatorname{Ker} \phi_{\hat{\ell}}$ that are maximal totally isotropic for the Weil form. This kernel is isomorphic to $(\mathbf{Z} / n \mathbf{Z}) \times(\mathbf{Z} / n \mathbf{Z})^{*}$, and the Weil form is given by $e\left(\left(x, x^{*}\right),\left(y, y^{*}\right)\right)=y^{*}(x)-x^{*}(y)$. Given a quotient group $\mathrm{H}$ of $\mathbf{Z} / n \mathbf{Z}$ and any homomorphism $u: \mathrm{H}^{*} \rightarrow \mathrm{H}$, the set of pairs $\left(x, x^{*}\right)$ in $(\mathbf{Z} / n \mathbf{Z}) \times \mathrm{H}^{*}$ such that the class of $x$ in $\mathrm{H}$ is $u\left(x^{*}\right)$ is such a subgroup, and they are all of this form. Their number is

$$
\sum_{\mathbf{Z} / n \mathbf{Z} \rightarrow \mathrm{H}}|\mathrm{H}|=\sum_{m \mid n} m=\sigma(n) .
$$

To each isogeny $\pi: \mathrm{JC} \rightarrow \mathrm{A}$ correspond $n^{2}$ curves in of genus 2 in $|\mathrm{L}|$, to wit the curves $\pi(\widetilde{\mathrm{C}})+x$, for each $x \in \operatorname{Ker} \phi_{\ell}$. So we get a total of $n^{2} \sigma(n)$ such curves, and they are all distinct. 
Proposition 2.2.- Assume each curve with class $\ell$ is integral; then $e\left(\mathrm{~J}^{d}(\mathrm{~A})\right)=n^{3} \sigma(n)$.

\section{A degeneration of $\mathrm{J}^{n-2}(\mathrm{~A})$}

Our aim is to relate the symplectic variety $\mathrm{J}^{d}(\mathrm{~A})$ constructed above with the generalized Kummer variety $\mathrm{K}_{n-1}(\mathrm{~A})$. Contrary to the case of $\mathrm{K} 3$ surfaces, these varieties do not seem to be birational for general A (except when $n=2$ ). However, we will prove that it is the case when $\mathrm{A}$ is a product of elliptic curves and $d=n-2$. For this, we will use the Mukai-Fourier transform for sheaves on A.

For any sheaf $\mathrm{F}$ on $\mathrm{A}$, we denote by $\mathcal{F}^{\bullet} \mathrm{F}$ the cohomology sheaves of the MukaiFourier transform of $\mathrm{F}$ (see ([M2]). If only $\mathcal{F}^{j} \mathrm{~F}$ is non-zero, we say that $\mathrm{F}$ has weak index $j$, and we write $\hat{\mathrm{F}}=\mathcal{F}^{j} \mathrm{~F}$; in that case, $\hat{\mathrm{F}}$ has weak index $2-j$, and $\hat{\mathcal{F}} \hat{\mathrm{F}} \simeq(-1)^{*} \mathrm{~F}$ (loc.cit., cor. 2.4). If $\mathrm{H}^{i}\left(\mathrm{~A}, \mathrm{~F} \otimes \mathrm{P}_{\hat{x}}\right)=0$ for all $\hat{x} \in \hat{\mathrm{A}}$ and all $i \neq j$, we say that $\mathrm{F}$ has index $j$; it implies that $\mathrm{F}$ has weak index $j$.

For any $\hat{x}$ in $\hat{\mathrm{A}}$, we denote by $\mathrm{P}_{\hat{x}}$ the corresponding line bundle on $\mathrm{A}$; we identify the dual of $\hat{\mathrm{A}}$ with $\mathrm{A}$, so that, for any $x$ in $\mathrm{A}, \mathrm{P}_{x}$ is a line bundle on $\hat{\mathrm{A}}$.

Let $\mathcal{L}$ be a sheaf on A corresponding to a point of $\overline{\mathcal{J}}^{d} \mathcal{C}$ with smooth support $\mathrm{C}$. For $\mathcal{L}$ generic in $\mathrm{J}^{d} \mathrm{C}$, the surface $\mathcal{L} \otimes \mathrm{Pic}^{0}(\mathrm{~A})$ does not meet the subvariety $\mathrm{W}_{d}(\mathrm{C})$ of $\mathrm{J}^{d} \mathrm{C}$, as soon as $g(\mathrm{C})>2+d$, i.e. $d<n-1$. In that case, one has $\mathrm{H}^{0}\left(\mathrm{~A}, \mathcal{L} \otimes \mathrm{P}_{\hat{x}}\right)=0$ for all $\hat{x}$ in $\hat{\mathrm{A}}$, so that $\mathcal{L}$ has index 1 , and $\hat{\mathcal{L}}$ is a locally free simple sheaf on $\hat{\mathrm{A}}$ of rank $n-d$, first Chern class $\hat{\ell}$ and Euler characteristic 0 .

Proposition 3.1.- Assume that the Néron-Severi group of A is generated by $\ell$. For $d<n-1$ and $\mathcal{L}$ generic in $\overline{\mathcal{J}}^{d} \mathcal{C}$, the vector bundle $\hat{\mathcal{L}}$ on $\hat{\mathrm{A}}$ is $\hat{\ell}$-stable.

Proof. We follow [FL] : assume $\hat{\mathcal{L}}$ is not stable, and look at torsion-free non-zero quotients of $\hat{\mathcal{L}}$ of smallest degree, and among those, pick one, Q, of smallest rank. Because $\mathrm{NS}(\hat{\mathrm{A}})=\mathbf{Z} \hat{\ell}$, the degree of $\mathrm{Q}$ is non-positive. The proofs of lemmes 2 and 3 of [FL] apply without change : $\mathrm{Q}$ has index 1 and if $\mathrm{K}$ be the kernel of $\hat{\mathcal{L}} \rightarrow \mathrm{Q}$, the sheaf $\mathcal{F}^{2} \mathrm{~K}$ has finite support. Consider the exact sequence

$$
0 \rightarrow \mathcal{F}^{1} \mathrm{~K} \rightarrow(-1)^{*} \mathcal{L} \rightarrow \hat{\mathrm{Q}} \rightarrow \mathcal{F}^{2} \mathrm{~K} \rightarrow 0
$$

since $c_{1}(\hat{\mathrm{Q}}) \cdot \ell=c_{1}(\mathrm{Q}) \cdot \hat{\ell} \leq 0([\mathrm{FL}]$, lemme 1$)$, the torsion sheaf $\hat{\mathrm{Q}}$ has finite support, hence index 0 . But this index is also $2-$ ind $Q=1$; this contradiction proves the proposition.

For each $d<n-1$, we have constructed a birational rational map between $\overline{\mathcal{J}}^{d} \mathcal{C}$ and an irreducible component $\mathcal{M}_{\hat{\mathrm{A}}}^{0}(n-d, \hat{\ell}, 0)$ of the moduli space $\mathcal{M}_{\hat{\mathrm{A}}}(n-d, \hat{\ell}, 0)$ of $\hat{\ell}$ semi-stable sheaves on $\hat{\mathrm{A}}$ of rank $n-d$, first Chern class $\hat{\ell}$ and Euler characteristic 0 . This map is a morphism if $d<0$. Let us interpret the maps $\alpha$ and $\beta$ in this context. Let $(\mathrm{C}, \mathcal{L})$ be a pair corresponding to a point of $\overline{\mathcal{J}}^{d} \mathcal{C}$; it follows from the exact sequence $0 \rightarrow \mathcal{O}_{\mathrm{C}} \rightarrow \mathcal{O}_{\mathrm{C}}(x) \rightarrow \mathbf{C}_{x} \rightarrow 0$ that $\operatorname{det} \widehat{\mathcal{O}_{\mathrm{C}}(x)} \simeq \operatorname{det} \widehat{\mathcal{O}_{\mathrm{C}}} \otimes \mathrm{P}_{-x}$, hence

$$
\operatorname{det} \hat{\mathcal{L}} \simeq \operatorname{det} \widehat{\mathcal{O}_{\mathrm{C}}} \otimes \mathrm{P}_{-\beta(\mathcal{L})} \simeq \operatorname{det} \widehat{\mathcal{O}_{\mathrm{A}}(-\mathrm{C})} \otimes \mathrm{P}_{-\beta(\mathcal{L})}
$$

Hence, the fibers of $(\alpha, \beta)$ are also the fibers of the map $\overline{\mathcal{J}}^{d} \mathcal{C} \rightarrow \operatorname{Pic}^{\ell}(\mathrm{A}) \times \operatorname{Pic}^{-\hat{\ell}}(\hat{\mathrm{A}})$ which sends $\mathcal{L}$ to $\left(\operatorname{det} \mathcal{L}, \operatorname{det} \mathcal{F}^{\bullet} \mathcal{L}\right)$. Let $\gamma: \mathcal{M}_{\hat{\mathrm{A}}}^{0}(n-d, \hat{\ell}, 0) \rightarrow \operatorname{Pic}^{-\ell}(\mathrm{A}) \times \operatorname{Pic}^{\hat{\ell}}(\hat{\mathrm{A}})$ be the map $\mathrm{E} \mapsto\left(\operatorname{det} \mathcal{F}^{\bullet} \mathrm{E}\right.$, $\left.\operatorname{det} \mathrm{E}\right)$, and let $\mathrm{M}_{n-d}(\hat{\mathrm{A}})$ be a fiber. We have proved the following.

Proposition 3.2.- Assume that the Néron-Severi group of A is generated by $\ell$. For $d<n-1$, the Fourier-Mukai transform induces a birational isomorphism between $\overline{\mathcal{J}}^{d} \mathcal{C}$ and an irreducible component of $\mathcal{M}_{\hat{\mathrm{A}}}(n-d, \hat{\ell}, 0)$ which sends $\mathrm{J}^{d}(\mathrm{~A})$ onto $\mathrm{M}_{n-d}(\hat{\mathrm{A}})$. 
We will now study the case where $n-d=2$ and $\mathrm{A}$ is the product of two general elliptic curves $\mathrm{F}$ and $\mathrm{G}$, with $\ell$ of bidegree $(1, n)$. One has $\hat{\mathrm{A}}=\hat{\mathrm{F}} \times \hat{\mathrm{G}}$, and $\hat{\ell}$ has bidegree $(n, 1)$. To avoid non-stable semi-stable sheaves, we will study the moduli space $\mathcal{M}_{\hat{\mathrm{A}}}^{\prime}$ of rank 2 sheaves on $\hat{A}$ with first Chern class $\hat{\ell}$ and Euler characteristic 0 which are semistable for the polarization $\hat{\ell}^{\prime}$ of bidegree $(n+1,1)$, and call $\mathrm{M}^{\prime}(\hat{\mathrm{A}})$ a fiber of the map $\gamma: \mathcal{M}_{\hat{\mathrm{A}}}^{\prime} \rightarrow \operatorname{Pic}^{-\ell}(\mathrm{A}) \times \operatorname{Pic}^{\hat{\ell}}(\hat{\mathrm{A}})$ defined above.

Proposition 3.3.- The moduli space $\mathcal{M}_{\hat{\mathrm{A}}}^{\prime}$ is smooth and birational to $\hat{\mathrm{A}}^{(n)} \times \mathrm{A}$. The variety $\mathrm{M}^{\prime}(\hat{\mathrm{A}})$ is smooth and birational to $\mathrm{K}_{n-1}(\hat{\mathrm{A}})$.

Proof. Let E be an $\hat{\ell}^{\prime}$-semi-stable rank 2 torsion free sheaf on $\hat{\mathrm{A}}$ with first Chern class $\hat{\ell}$ and Euler characteristic 0 . Let $x \in \mathrm{A}$; by semi-stability of $\mathrm{E}^{*}$, one has $\mathrm{H}^{2}\left(\hat{\mathrm{A}}, \mathrm{E} \otimes \mathrm{P}_{x}^{-1}\right)=0$, hence $h^{0}\left(\hat{\mathrm{A}}, \mathrm{E} \otimes \mathrm{P}_{x}^{-1}\right)=h^{1}\left(\hat{\mathrm{A}}, \mathrm{E} \otimes \mathrm{P}_{x}^{-1}\right)$. Since $\hat{\mathcal{F}} \mathrm{E}$ is non-zero, for at least one $x$, these numbers are non-zero and there is an inclusion $\mathrm{P}_{x} \hookrightarrow \mathrm{E}$; let $\mathrm{K}$ be the kernel of $\mathrm{E} \rightarrow \mathrm{E} / \mathrm{P}_{x} \rightarrow\left(\mathrm{E} / \mathrm{P}_{x}\right) /\left(\mathrm{E} / \mathrm{P}_{x}\right)_{\text {tors }}$. There is an exact sequence

$$
0 \rightarrow \mathrm{K} \rightarrow \mathrm{E} \rightarrow \mathcal{I}_{\mathrm{Z}} \otimes \mathrm{K}^{\prime} \rightarrow 0
$$

where $\mathrm{K}^{\prime}$ is a line bundle. The line bundle $\mathrm{K}$ has bidegree $(a, b)$, with $a$ and $b$ non-negative and $b(n+1)+a \leq(2 n+1) / 2$ (by $\hat{\ell}^{\prime}$-semi-stability); hence $b=0$ and $\mathrm{Z}$ is a subscheme of $\hat{\mathrm{A}}$ of length $n-a$.

Set $\mathrm{M}=\mathrm{K}^{\prime} \otimes \mathrm{K}^{-1}$. By Serre duality, $\operatorname{Ext}_{\hat{\mathrm{A}}}^{1}\left(\mathcal{I}_{\mathrm{Z}} \otimes \mathrm{K}^{\prime}, \mathrm{K}\right)$ and $\mathrm{H}^{1}\left(\hat{\mathrm{A}}, \mathcal{I}_{\mathrm{Z}} \otimes \mathrm{M}\right)^{*}$ are isomorphic. Assume $\mathrm{H}^{0}\left(\hat{\mathrm{A}}, \mathcal{I}_{\mathrm{Z}} \otimes \mathrm{M}\right)=0$, one has

$$
h^{1}\left(\hat{\mathrm{A}}, \mathcal{I}_{\mathrm{Z}} \otimes \mathrm{M}\right)=\operatorname{length}(\mathrm{Z})-\chi(\hat{\mathrm{A}}, \mathrm{M})=a
$$

and $a>0$ (otherwise $\mathcal{I}_{\mathrm{Z}} \otimes \mathrm{K}^{\prime}$ would be a subsheaf of $\mathrm{E}$ with $\hat{\ell}^{\prime}$-slope $2 n+1$ ), and $\mathrm{E}$ depends on at most $2 n+3-a$ parameters $\left(2\right.$ for $\mathrm{K}, 2$ for $\mathrm{K}^{\prime}, 2(n-a)$ for $\mathrm{Z}$ and $a-1$ for the extension). Since each component of $\mathcal{M}_{\hat{\mathrm{A}}}^{\prime}$ has dimension $2 n+2$, this forces $a=1$ for E generic. Let $\mathcal{M}^{0}$ be the subset of $\mathcal{M}_{\hat{\mathrm{A}}}^{\prime}$ parametrized in this fashion.

Assume now $\mathrm{H}^{0}\left(\hat{\mathrm{A}}, \mathcal{I}_{\mathrm{Z}} \otimes \mathrm{M}\right) \neq 0$; one checks (by projecting onto $|\mathrm{M}|$ ), that the set of pairs $(\mathrm{Z}, \mathrm{D})$ with $\mathrm{D} \in|\mathrm{M}|$ and $\mathrm{Z} \subset \mathrm{D}$, has dimension $\leq n-2 a-1+n-a$. Hence $\mathrm{E}$ depends on at most $2 n-3 a-1-\chi\left(\hat{\mathrm{A}}, \mathcal{I}_{\mathrm{Z}} \otimes \mathrm{M}\right)+4=2 n-2 a+3$ parameters. For $\mathrm{E}$ generic, this forces $a=0, \mathrm{Z}$ reduced and $h^{0}\left(\hat{\mathrm{A}}, \mathcal{I}_{\mathrm{Z}} \otimes \mathrm{M}\right)=1$. This yields a component of $\mathcal{M}_{\hat{\mathrm{A}}}^{\prime}$ which can be parametrized as follows. Let $\mathrm{Z}=\left(\hat{f}_{1}, \hat{g}_{1}\right)+\ldots+\left(\hat{f}_{n}, \hat{g}_{n}\right)$ be generic in $\hat{\mathrm{A}}^{(n)}$, set $\mathrm{L}=\mathcal{O}_{\hat{\mathrm{F}}}\left(\hat{f}_{1}+\ldots+\hat{f}_{n}\right)$, and let $f \in \mathrm{F}$ and $\hat{g} \in \hat{\mathrm{G}}$. The vector space $\operatorname{Ext}_{\hat{\mathrm{A}}}^{1}\left(\mathcal{I}_{\mathrm{Z}} \otimes p_{\hat{\mathrm{F}}}^{*} \mathrm{~L} \otimes p_{\hat{\mathrm{G}}}^{*} \mathcal{O}_{\hat{\mathrm{G}}}(\hat{g}), \mathcal{O}_{\hat{\mathrm{A}}}\right)$ has dimension 1 , hence there is a unique extension

$$
0 \rightarrow p_{\hat{\mathrm{F}}}^{*} \mathrm{P}_{f} \rightarrow \mathrm{E} \rightarrow \mathcal{I}_{\mathrm{Z}} \otimes p_{\hat{\mathrm{F}}}^{*}\left(\mathrm{~L} \otimes \mathrm{P}_{f}\right) \otimes p_{\hat{\mathrm{G}}}^{*} \mathcal{O}_{\hat{\mathrm{G}}}(\hat{g}) \rightarrow 0
$$

where $\mathrm{E}$ is locally free (it satisfies the Cayley-Bacharach condition; see for example th. 5.1.1 of $[\mathrm{HL}])$ and stable (the only thing to check is $\mathrm{H}^{0}\left(\hat{\mathrm{A}}, \mathrm{E} \otimes p_{\hat{\mathrm{F}}}^{*} \mathrm{P}_{-f} \otimes p_{\hat{\mathrm{G}}}^{*} \mathcal{O}_{\hat{\mathrm{G}}}(-\hat{g})\right)=0$, and this is true because the extension is non-trivial). This yields a rational map

$$
\phi: \hat{\mathrm{A}}^{(n)} \times \mathrm{F} \times \hat{\mathrm{G}} \rightarrow-\rightarrow \mathcal{M}_{\hat{\mathrm{A}}}^{\prime}
$$

which is birational onto its image : given a locally free $\mathrm{E}$ as above, one recovers $f$ and the $\left(\hat{g}_{i}-\hat{g}\right)$ 's by noting that the set $\mathrm{C}_{\mathrm{E}}=\left\{x \in \mathrm{A} \mid \mathrm{H}^{0}\left(\hat{\mathrm{A}}, \mathrm{E} \otimes \mathrm{P}_{x}\right) \neq 0\right\}$ is

$$
(\{-f\} \times \mathrm{G}) \cup \bigcup_{i=1}^{n}\left(\mathrm{~F} \times\left\{\left[\mathcal{O}_{\hat{\mathrm{G}}}\left(\hat{g}_{i}-\hat{g}\right)\right]\right\}\right),
$$


the $\hat{f}_{i}$ 's because $\operatorname{Ext}_{\hat{\mathrm{A}}}^{1}\left(\mathcal{I}_{\mathrm{Z}} \otimes p_{\hat{\mathrm{F}}}^{*} \mathrm{~L} \otimes p_{\hat{\mathrm{G}}}^{*} \mathcal{O}_{\hat{\mathrm{G}}}(\hat{g}), \mathcal{O}_{\hat{\mathrm{A}}}\right)$ must be non-zero, and $\hat{g}$ by noting that $\operatorname{det} \mathrm{E} \simeq p_{\hat{\mathrm{F}}}^{*}\left(\mathrm{~L} \otimes \mathrm{P}_{2 f}\right) \otimes p_{\hat{\mathrm{G}}}^{*} \mathcal{O}_{\hat{\mathrm{G}}}(\hat{g})$. Because $\mathrm{H}^{0}\left(\hat{\mathrm{A}}, \mathrm{E} \otimes p_{\hat{\mathrm{F}}}^{*}\left(\mathrm{P}_{-f} \otimes \mathcal{O}_{\hat{\mathrm{F}}}\left(-f_{1}\right)\right) \otimes p_{\hat{\mathrm{G}}}^{*} \mathcal{O}_{\hat{\mathrm{G}}}\left(\hat{g}_{1}-\hat{g}\right)\right)$ is non-zero, there exists an exact sequence $(*)$ with $\mathrm{K}$ of bidegree $(1,0)$. This proves that the set $\mathcal{M}^{0}$ defined above is contained in the image of $\phi$, which must therefore be $\mathcal{M}_{\hat{\mathrm{A}}}^{\prime}$.

Finally, E has weak index $1, \mathcal{F}^{1} \mathrm{E}$ has support on $\mathrm{C}_{\mathrm{E}}$, and fixing $\operatorname{det} \mathcal{F}^{1} \mathrm{E}$ amounts to fixing $\left[\mathcal{O}_{\mathrm{A}}\left(\mathrm{C}_{\mathrm{E}}\right)\right]$. It follows that taking a fiber of $\gamma$ amounts to fixing $f, \sum\left(\hat{g}_{i}-\hat{g}\right), \sum \hat{f}_{i}$ and $\hat{g}$; hence $\mathrm{M}^{\prime}(\hat{\mathrm{A}})$ is birational to $\mathrm{K}_{n-1}(\hat{\mathrm{A}})$.

The following proof is due to D. Huybrechts, and uses ideas from prop. 2.2 of [GH].

Theorem 3.4.- Let $(\mathrm{A}, \ell)$ be a polarized abelian surface of type $(1, n)$ whose Néron-Severi group is generated by $\ell$. The symplectic varieties $\mathrm{J}^{n-2}(\mathrm{~A}), \mathrm{M}_{2}(\hat{\mathrm{A}})$ and $\mathrm{K}_{n-1}(\hat{\mathrm{A}})$ are deformation equivalent. In particular, they are all irreducible symplectic.

Proof. Let $f: \hat{\mathcal{A}} \rightarrow \mathrm{S}$ be a family of polarized abelian surfaces, where $\mathrm{S}$ is smooth quasiprojective, with a relative polarization $\hat{\mathcal{L}}$ of type $(1, n)$, such that the fiber over a point $0 \in \mathrm{S}$ is $\hat{\mathrm{F}} \times \hat{\mathrm{G}}$ with a polarization of bidegree $(n, 1)$; assume also that the Néron-Severi group of a very general fiber of $f$ has rank 1 . Let $g: \mathcal{M} \rightarrow \mathrm{S}$ be the (projective) relative moduli space of $\hat{\mathcal{L}}$-semi-stable sheaves of rank 2 with first Chern class $\hat{\ell}$ and Euler characteristic 0 on the fibers of $f$ ( $c f$. [HL], th. 4.3.7, p. 92).

Lemma 3.5.- Under the hypothesis of the proposition, any rank 2 torsion free sheaf on $\hat{\mathrm{A}}$ with first Chern class $\hat{\ell}$ which is either simple or semi-stable is stable.

Proof. Assume that a sheaf E with these numerical characters is not stable. There exists an exact sequence

$$
0 \rightarrow \mathrm{K} \rightarrow \mathrm{E} \rightarrow \mathcal{I}_{\mathrm{Z}} \otimes \mathrm{K}^{\prime} \rightarrow 0
$$

where $\mathrm{K}$ and $\mathrm{K}^{\prime}$ are line bundles on $\hat{\mathrm{A}}$ with $c_{1}(\mathrm{~K})=k \hat{\ell}, c_{1}\left(\mathrm{~K}^{\prime}\right)=(1-k) \hat{\ell}$ and $k>0$. This proves that $\mathrm{E}$ is not semi-stable; moreover, $\mathrm{K} \otimes \mathrm{K}^{\prime-1}$ is ample, hence there exists a non-zero morphism $u: \mathrm{K}^{\prime} \rightarrow \mathrm{K}$, which induces an endomorphism $\mathrm{E} \rightarrow \mathcal{I}_{\mathrm{Z}} \otimes \mathrm{K}^{\prime} \stackrel{u}{\rightarrow} \mathrm{K} \rightarrow \mathrm{E}$ which is not a homothety, and $\mathrm{E}$ is not simple.

By the lemma, the (closed) locus of non-stable points in $\mathcal{M}$ does not project onto $\mathrm{S}$. By replacing $\mathrm{S}$ with an open subset, we may assume that there are no such points. Let now $\mathcal{S} \rightarrow \mathrm{S}$ be the (smooth) relative moduli space of simple sheaves on the fibers of $f$ (see $[\mathrm{AK}])$. There are embeddings $\mathcal{M} \subset \mathcal{S}$ and $\mathcal{M}_{\hat{\mathrm{F}} \times \hat{\mathrm{G}}}^{\prime} \subset \mathcal{S}_{0}$ as closed and open subsets. Let $\mathcal{S}^{\prime}=\mathcal{S}-\left(\mathcal{S}_{0}-\mathcal{M}_{\hat{\mathrm{F}} \times \hat{\mathrm{G}}}^{\prime}\right)$; it is open in $\mathcal{S}$, hence smooth over $\mathrm{S}$. Let $\mathcal{M}^{\prime}$ be the closure of $g^{-1}(\mathrm{~S}-\{0\})$ in $\mathcal{S}^{\prime} ;$ the fibers of $g^{\prime}: \mathcal{M}^{\prime} \rightarrow \mathrm{S}$ are projective off 0 , and contained in $\mathcal{M}_{\hat{\mathrm{F}} \times \hat{\mathrm{G}}}^{\prime}$ over 0 . Norton's criterion $([\mathrm{N}])$ shows that points in $\mathcal{M}_{0}^{\prime}$ are separated in the moduli space of simple sheaves on $\hat{\mathcal{A}}$ (because they are stable), hence in $\mathcal{S}$; therefore, $\mathcal{M}^{\prime}$ is separated. By semi-continuity, $\mathcal{M}_{0}^{\prime}$ is a closed subset of $\mathcal{M}_{\hat{\mathrm{F}} \times \hat{\mathrm{G}}}^{\prime}$ of the same dimension, hence they are equal. Using the lemma, we get, after shrinking $\mathrm{S}$ again, a proper family $g^{\prime}: \mathcal{M}^{\prime} \rightarrow \mathrm{S}$ with projective irreducible smooth fibers which coincide with $g: \mathcal{M} \rightarrow \mathrm{S}$ off 0 .

By prop. 3.2, $\mathrm{J}^{n-2}(\mathrm{~A})$ is birationally isomorphic to $\mathrm{M}_{2}(\hat{\mathrm{F}} \times \hat{\mathrm{G}})$, and we just saw that the latter deforms to $\mathrm{M}^{\prime}(\hat{\mathrm{A}})$, itself birationally isomorphic to $\mathrm{K}_{n-1}(\hat{\mathrm{F}} \times \hat{\mathrm{G}})$ by prop. 3.3 ; in particular, these symplectic varieties are all irreducible symplectic. Since birationally isomorphic smooth projective irreducible symplectic varieties are deformation equivalent $([\mathrm{H}]$, th. 10.12), the theorem is proved. 
Corollary 3.6.- Let $(\mathrm{A}, \ell)$ be a general polarized abelian surface of type $(1, n)$. The moduli space $\mathcal{M}_{\mathrm{A}}(2, \ell, 0)$ is smooth irreducible.

Corollary ([GS]) 3.7.- Let A be an abelian surface. The Euler characteristic of $\mathrm{K}_{n-1}(\mathrm{~A})$ is $n^{3} \sigma(n)$.

\section{REFERENCES}

[AK] A. Altman, S. Kleiman : Compactifying the Picard Scheme, Adv. Math. 35 (1980), 50-112.

[B1] A. Beauville : Counting rational curves on K3 surfaces, e-print alg-geom 9701019.

[B2] A. Beauville : Variétés kähleriennes dont la première classe de Chern est nulle, J. Diff. Geom. 18 (1983), 755-782.

[BL] J. Bryan, N.C. Leung : The Enumerative Geometry of K3 Surfaces and Modular Forms, e-print alg-geom 9711031.

[FL] R. Fahlaoui, Y. Laszlo : Transformée de Fourier et stabilité sur les surfaces abéliennes, Comp. Math. 79 (1991), 271-278.

[G] L. Göttsche : A conjectural generating function for numbers of curves on surfaces, e-print alg-geom 9711012 .

[GS] L. Göttsche, W. Soergel : Perverse sheaves and the cohomology of Hilbert schemes of smooth algebraic surfaces, Math. Ann. 296 (1993), 235-245.

[GH] L. Göttsche, D. Huybrechts : Hodge Numbers of Moduli Spaces of Stable Bundles on K3 surfaces, Intern. J. Math. 7 (1996), 359-372.

[H] D. Huybrechts : Compact Hyperkähler Manifolds, Habilitationsschrift, Universität-GH Essen, 1997.

[HL] D. Huybrechts, M. Lehn : The Geometry of Moduli Spaces of Sheaves, Aspects of Mathematics, Vieweg, Braunschweig/Wiesbaden, 1997.

[M1] S. Mukai : Symplectic structure of the moduli space of sheaves on an abelian or K3 surface, Invent. Math. 77 (1984), 101-116.

[M2] S. Mukai : Duality between $\mathrm{D}(\mathrm{X})$ and $\mathrm{D}(\hat{\mathrm{X}})$ with its application to Picard sheaves, Nagoya Math. J. 81 (1981), 153-175.

[N] A. Norton : Analytic moduli of complex vector bundles, Indiana Univ. Math. J. 28 (1979), 365-387.

[YZ] S.-T. Yau, E. Zaslow : BPS states, string duality, and nodal curves on K3, Nuclear Physics B 471 (1996), 503-512; also preprint hep-th 9512121.

Olivier DEBARRE

IRMA - Mathématique - CNRS

Université Louis Pasteur

7, rue René Descartes

67084 Strasbourg Cédex - France

Adresse électronique : debarre@math.u-strasbg.fr 